\title{
Colchicine, an anti-rheumatic agent, as a potential compound for the treatment of COVID-19
}

\author{
Carlo Perricone, Elena Bartoloni, Roberto Gerli \\ Rheumatology Unit, Department of Medicine, University of Perugia, Italy
}

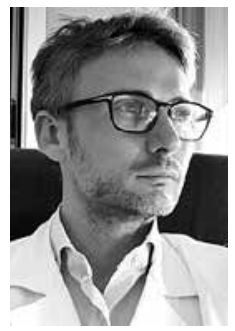

The outbreak of severe acute respiratory syndrome coronavirus 2 (SARS-CoV-2) has swept the world in a pandemic. The disease (COVID-19) is characterized by acute respiratory distress, and there is an immediate need for any effective agent before the development of a vaccine. Coronavirus-19 disease (COVID-19) is an infectious disease caused by SARS-CoV-2, a $\beta$-coronavirus which causes pneumonia, requires intensive care unit hospitalization in about $10 \%$ of cases and can lead to a fatal outcome [1].

To date, there is no registered molecule for the treatment of COVID-19 patients. However, there are ongoing trials on the use of antivirals and immunosuppressive or immunomodulating drugs that have demonstrated some efficacy towards COVID-19 [2]. Since preliminary in vitro experiments have suggested that antirheumatic drugs, chloroquine (CQ) and hydroxychloroquine (HCQ), have anti-viral activity, these agents are in the therapeutic armamentarium against COVID-19 [3]. Nonetheless, a fundamental role in pathogenesis of SARS-CoV-2 infection damage is played by monocytes/ macrophages [4].

In fact, infiltrating macrophages can promote hyperinflammation, characterized by increased levels of interleukin (IL)-6, tumor necrosis factor-alpha (TNF- $\alpha$ ), IL-1 $\beta$, ferritin and neutrophil/lymphocyte ratio, and subsequent possible lung fibrotic complications [5]. As well described, virus-induced activation of the NLRP3 inflammasome appears to play a key pathogenic role through IL-1 $\beta / \mathrm{IL}-18$ release, which is able to amplify monocyte/macrophage activation, but also to decrease type I interferon synthesis by infected lungs. Activated monocytes can also mediate microvascular thrombotic events through production of tissue factor and activation of the extrinsic coagulation pathway. Coagulopathy and microvascular damage are amplified by neutrophil recruitment, favoured by activated endothelial cells, and consequent release of neutrophil extracellular traps (NETS), and by complement activation associated with production of anaphylatoxin $\mathrm{C} 5 \mathrm{a}$ acting as further chemoattractant $[5,6]$.

Colchicine targets a wide range of mechanisms associated with monocyte/macrophage activation and beyond. It acts through inhibition of the NLRP3 inflammasome [7], thus reducing IL-1 $\beta / I L-18$ synthesis and induction of pro-inflammatory cytokines, inhibits neutrophil activation, migration [8] and generation of NETs [9], protects endothelial damage preventing microvascular thrombosis [8], and has complement inhibitor activity and anti-fibrotic properties. Moreover, colchicine has anti-viral properties against Flaviviridae and against the recombinant demyelinating strain of mouse hepatitis virus RSA59 as demonstrated by reducing virus replication. Indeed, microtubules are crucial in cell entry of Flaviviridae and colchicine can block neuronal transport of RSA59 [10, 11]. It inhibits respiratory syncytial virus (RSV) replication by reducing IL- 6 and TNF- $\alpha$ levels [12]. A rationale of colchicine derivatives as anti-HIV agents has been proposed too [13].

Concerning SARS-CoV, it was recently documented that the virus and its accessory protein are potent activators of pro-IL-1 $\beta$ gene transcription and protein maturation, and thus are able to activate the NLRP3 inflammasome [14].

Moreover, certain subsets of the 2003-SARS coronavirus replication machinery have been shown to move in the cell in a manner that corresponds with microtubule-associated transport, inducing the formation of double-membrane vesicles in infected cells. This may occur during SARS-CoV-2 infection and microtubule disruption may influence viral genome replication. Finally, dysregulated activation and inflammatory activity of myeloid cells is one the main pathogenic events characterizing the infection by coronaviruses $[15,16]$.

Colchicine is a well-known inhibitor of the proinflammatory mechanisms induced by neutrophils and 


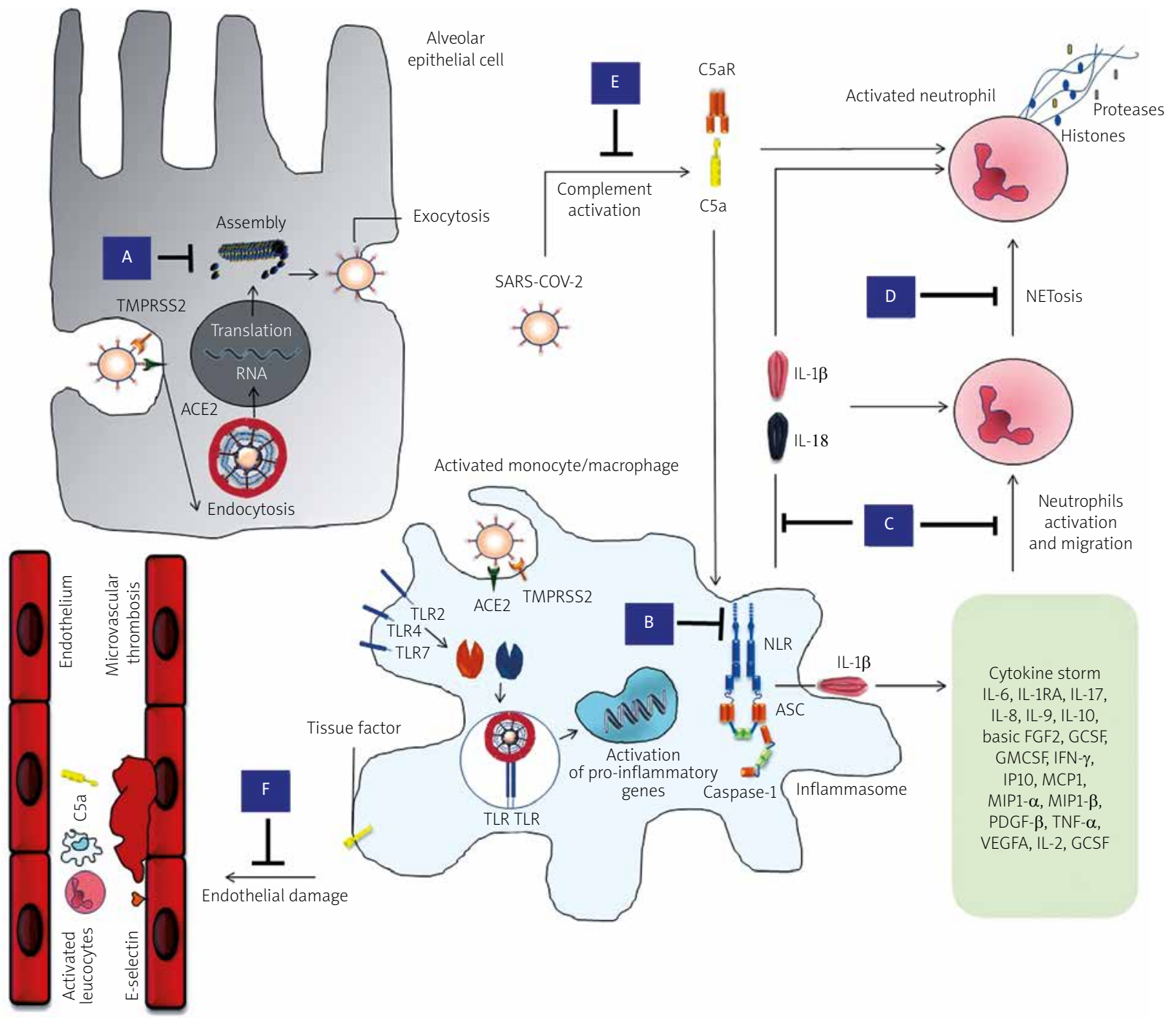

Fig. 1. Colchicine may be effective against SARS CoV-2 infection by acting through different mechanisms. Impairment of SARS-CoV-2 replication by disrupting microtubules, crucial in viral trafficking and formation of double-membrane vesicles (A). Potent inhibition of the NLRP3 inflammasome with decrease of IL-1 $\beta$ and consequent downstream production of several pro-inflammatory cytokines that are produced in excess in COVID-19 (cytokine storm) (B). Inhibition of neutrophil activation, migration and adhesion to endothelial cells through diminished expression of L-selectin (C). Inhibition of NET generation (D). Prevention of further leukocyte migration and microvascular thrombosis due to inhibition of complement activation via microtubule disruption, inhibition of $\mathrm{C} 5 \mathrm{a}$ and reduced expression of $\mathrm{C} 5 \mathrm{aR}$ (E). Prevention of endothelial damage by an indirect effect secondary to inhibition of excessive inflammatory activation and by impairment of E-selectin-mediated neutrophil adherence to pro-inflammatory cytokine-activated endothelial cells (F).

thus it was proposed as an adjuvant treatment for RSV bronchiolitis. Figure 1 summarizes the possible targets of colchicine during SARS-CoV-2 infection.

Thus, colchicine has broad anti-inflammatory effects and anti-viral properties, is not hampered by an immunosuppressant effect and is an inexpensive drug. The gastrointestinal disturbance that may be seen in up to $10 \%$ of patients usually does not require drug interrup- tion and should not limit its usage in COVID-19 patients. It has been suggested that colchicine may be trialled in an appropriate COVID-19 patient population to reduce both viral entry and inflammatory status. Increasing successful case reports have been reported with colchicine usage in the context of the COVID-19 pandemic [17-24].

We designed two trials approved by the Italian Drug Agency that are currently ongoing to verify the effect 
of this agent in the early/intermediate phases of the disease (EudraCT2020-001806-42, NCT04375202), and similar investigations are ongoing in other countries (NCT04322565, NCT04355143, NCT04360980, NCTO4350320, NCT04326790, NCT04328480, NCT04367168, NCT04363437, NCT04322682).

Deftereos et al. [25] provided so far the largest contribution from a prospective, open-label, randomized clinical trial (the Greek Study in the Effects of Colchicine in COVID-19 Complications Prevention - GRECCO-19) on 105 patients hospitalized with COVID-19 randomized $1: 1$ to either standard medical treatment or colchicine with standard medical treatment. They evaluated 50 (47.6\%) patients in the control group and 55 (52.4\%) in the colchicine group. The primary end point (including the maximum high-sensitivity cardiac troponin level; time for C-reactive protein to reach more than 3 times the upper reference limit; and time to deterioration by 2 points on a 7-grade clinical status scale) rate was $14.0 \%$ in the control group and $1.8 \%$ in the colchicine group. The event-free survival time was similar between the two groups (18.6 [0.83] days the in the control group vs. 20.7 [0.31] in the colchicine group), as was the frequency of adverse events. The overall results suggest a clinical benefit of colchicine in time to deterioration and possibly a cardioprotective effect.

To conclude, although no conclusive results can still be drawn, if the effectiveness of colchicine is confirmed, this agent may extremely useful in the treatment of COVID-19, particularly due to its recognized safety and marginal cost.

The authors declare no conflict of interest.

\section{References}

1. Remuzzi A, Remuzzi G. COVID-19 and Italy: what next? Lancet 2020; 395: 1225-1228, DOI: 10.1016/S0140-6736(20)30627-9.

2. Stebbing J, Phelan A, Griffin I, et al. COVID-19: combining antiviral and anti-inflammatory treatments. Lancet Infect Dis 2020; 20: P400-402, DOI: 10.1016/S1473-3099(20)30132-8.

3. Perricone C, Triggianese P, Bartoloni E, et al. The anti-viral facet of anti-rheumatic drugs: Lessons from COVID-19. J Autoimmun 2020; 111: 102468, DOI: 10.1016/j.jaut.2020.102468.

4. Merad M, Martin JC. Pathological inflammation in patients with COVID-19: a key role for monocytes and macrophages. Nat Rev Immunol 2020; 20: 355-362, DOI: 10.1038/s41577-020-0331-4.

5. Risitano AM, Mastellos DC, Huber-Lang M, et al. Complement as a target in COVID-19? Nat Rev Immunol 2020; 20: 343-344, DOI: 10.1038/s41577-020-0320-7.

6. Siddiqi HK, Mehra MR. COVID-19 illness in native and immunosuppressed states: a clinical-therapeutic staging proposal. J Heart Lung Transplant 2020; 39: 405-407, DOI: 10.1016/ j.healun.2020.03.012.
7. Martinon F, Pétrilli V, Mayor A, et al. Gout-associated uric acid crystals activate the NALP3 inflammasome. Nature 2006; 440: 237-441, DOI: 10.1038/nature04516.

8. Nuki G. Colchicine: its mechanism of action and efficacy in crystal-induced inflammation. Curr Rheumatol Rep 2008; 10: 218-227, DOI: 10.1007/s11926-008-0036-3.

9. Apostolidou E, Skendros P, Kambas K, et al. Neutrophil extracellular traps regulate IL-1 $\beta$-mediated inflammation in familial Mediterranean fever. Ann Rheum Dis 2016; 75: 269-277, DOI: 10.1136/annrheumdis-2014-205958.

10. Richter M, Boldescu V, Graf D, et al. Synthesis, biological evaluation, and molecular docking of combretastatin and colchicine derivatives and their hCE1-Activated Prodrugs as antiviral agents. ChemMedChem 2019; 14: 469-483, DOI: 10.1002/cmdc. 201800641.

11. Biswas K, Das Sarma J. Effect of microtubule disruption on neuronal spread and replication of demyelinating and nondemyelinating strains of mouse hepatitis virus in vitro. J Virol 2014; 88: 3043-3047, DOI: 10.1128/JVI.02545-13.

12. Lu N, Yang Y, Liu H, et al. Inhibition of respiratory syncytial virus replication and suppression of RSV-induced airway inflammation in neonatal rats by colchicine. Biotech 2019; 9 : 392, DOI: 10.1007/s13205-019-1917-z.

13. Worachartcheewan A, Songtawee N, Siriwong S, et al. Rational design of colchicine derivatives as anti-HIV agents via QSAR and molecular docking. Med Chem 2019; 15: 328-340, DOI: 10.2174/1573406414666180924163756.

14. Chen IY, Moriyama M, Chang MF, Ichinohe T. Severe acute respiratory syndrome coronavirus viroporin $3 a$ activates the NLRP3 inflammasome. Front Microbiol 2019; 10: 50, DOI: 10.3389/fmicb.2019.00050.

15. Channappanavar R, Perlman S. Evaluation of activation and inflammatory activity of myeloid cells during pathogenic human coronavirus infection. Methods Mol Biol 2020; 2099: 195204, DOI: 10.1007/978-1-0716-0211-9_15.

16. Qin C, Zhou L, Hu Z, et al. Dysregulation of immune response in patients with COVID-19 in Wuhan, China. Clin Infect Dis 2020; 71: 762-768, DOI: 10.1093/cid/ciaa248.

17. Elshafei MN, Khalil A, El-Bardissy A, et al. The efficacy of colchicine in the management of coronavirus disease 2019: a protocol for systematic review and meta-analysis. Medicine (Baltimore) 2020; 99: e21911, DOI: 10.1097/MD.0000000000021911.

18. Della-Torre E, Ramirez GA, Dagna L, Tresoldi M. Colchicine treatment in community healthcare setting to prevent severe COVID-19. Ann Rheum Dis 2020, DOI: 10.1136/annrheumdis2020-218759 [Epub ahead of print].

19. Piantoni S, Colombo E, Furloni R, et al. Anti-inflammatory action of colchicine in hospitalised patients with COVID-19. Response to: 'Colchicine treatment in community healthcare setting to prevent severe COVID-19' by Della-Torre et al. Ann Rheum Dis 2020, DOI: 10.1136/annrheumdis-2020-218806 [Epub ahead of print].

20. Papadopoulos C, Patoulias D, Teperikidis E, et al. Colchicine as a potential therapeutic agent against cardiovascular complications of COVID-19: an exploratory review. SN Compr Clin Med 2020: 1-11, DOI: 10.1007/s42399-020-00421-x [Epub ahead of print]. 
21. Schlesinger N, Firestein BL, Brunetti L. Colchicine in COVID-19: an old drug, new use. Curr Pharmacol Rep 2020: 1-9, DOI 10.1007/s40495-020-00225-6 [Epub ahead of print].

22. Scarsi M, Piantoni S, Colombo E, et al. Association between treatment with colchicine and improved survival in a single- centre cohort of adult hospitalised patients with COVID-19 pneumonia and acute respiratory distress syndrome. Ann Rheum Dis 2020 79: 1286-1289, DOI: 10.1136/annrheumdis-2020-217712.

23. Mansouri N, Marjani M, Tabarsi P, et al. Successful treatment of Covid-19 associated cytokine release syndrome with colchicine. A case report and review of literature. Immunol Invest
2020: 1-7, DOI: 10.1080/08820139.2020.1789655 [Epub ahead of print].

24. Gandolfini I, Delsante M, Fiaccadori E, et al. COVID-19 in kidney transplant recipients. Am J Transplant 2020; 20: 1941-1943, DOI: 10.1111/ajt.15891.

25. Deftereos SG, Giannopoulos G, Vrachatis DA, et al. Effect of colchicine vs standard care on cardiac and inflammatory biomarkers and clinical outcomes in patients hospitalized with coronavirus disease 2019: the GRECCO-19 randomized clinical trial. JAMA Netw Open 2020; 3: e2013136, DOI: 10.1001/jamanetworkopen.2020.13136. 